\title{
Estudio del Comportamiento I vs. V de la Descarga Luminiscente de Baja Presión en Corriente Continua y Corriente Continua Pulsante en un Reactor Tipo Calorímetro
}

\author{
Low Pressure Glow Discharge I vs. V Behavior Study, in a DC and Pulsed DC in \\ a Calorimeter Type Reactor
}

J. F. Cepeda Grimaldos ${ }^{\mathrm{a}, *}$

A. Sarmiento Santos ${ }^{\mathrm{a}}$

I. Supelano García ${ }^{\mathrm{a}}$

Recepción: 22-feb-13

Aceptación: 27-mar-13

\begin{abstract}
Resumen
Al generar una descarga luminiscente con corriente continua o corriente continua pulsada en diferentes atmósferas gaseosas, ésta se puede caracterizar por el color o por el comportamiento de la curva de I vs. V donde, para cada una de las atmósferas se pueden presentar los tipos de régimen de la descarga luminiscente, que son: descarga luminiscente subnormal, descarga luminiscente normal y descarga luminiscente anormal. En este trabajo se generaron estos tipos de descargas en un reactor cilíndrico concéntrico cuyo electrodo interno hace las veces de cátodo, y el externo, de ánodo. Entre los dos electrodos fue generada la descarga luminiscente de baja presión, utilizando diferentes atmósferas gaseosas (aire, argón e hidrógeno). Las descargas fueron estudiadas a varias presiones, entre 1 torr y 6 torr, y también a diferentes corrientes, entre $200 \mathrm{~mA}$ y $500 \mathrm{~mA}$, con incremento de $50 \mathrm{~mA}$ en los regímenes de corriente continua y corriente continua pulsada. Las características de dichas descargas fueron analizadas a través de sus curvas I vs. V.
\end{abstract}

Palabras clave: Curvas I vs. V, Descarga luminiscente subnormal, Descarga luminiscente normal, Descarga luminiscente anormal.

\begin{abstract}
When a glow discharge is generated with a DC, or pulsating a DC power at diverse atmospheres, it can be characterized by its color or by I vs. V curve behavior. For various atmospheres it can be presented the different glow discharge types, such as: a subnormal glow discharge, a normal glow discharge and an abnormal glow discharge. In this paper, these glow discharge types are generated, in a concentric cylindrical reactor, in which its internal cylinder works as the cathode and the external cylinder as the anode.
\end{abstract}

${ }^{a}$ GSEC, Escuela de Física, Facultad de Ciencias Básicas, Universidad Pedagógica y Tecnológica de Colombia, Tunja, Boyacá.

*Autor de correspondencia: asarmiento.santos@uptc.edu.co 
Between the electrodes, it was generated a low pressure glow discharge, through various gaseous atmospheres (air, argon and hydrogen). Such discharges were studied at various pressures values between 1 torr and 6 torr and at various currents values, between $200 \mathrm{~mA}$ and $500 \mathrm{~mA}$, with a $50 \mathrm{~mA}$ increment for each current variation. For this, two current types were utilized: DC and pulsating DC. The discharge characteristics were analyzed by its I vs. V curves.

Key words: I vs. V Curves, Subnormal Glow Discharge, Normal Glow Discharge, Abnormal Glow Discharge

\section{Introducción}

La descarga luminiscente de baja presión tiene gran variedad de aplicaciones tecnológicas [1]; por ello es indispensable crear dispositivos especiales que permitan generarla con miras a aplicaciones particulares, haciendo acopio del cúmulo de trabajos experimentales y teóricos existentes [2].

Se sabe que cualquier gas en condiciones normales contiene un determinado número de electrones e iones. A nivel del mar, la atmósfera contiene, en promedio, unos 1000 iones positivos y negativos por centímetro cúbico, debido a la radiación cósmica, ultravioleta y a la radio-actividad. En ausencia de campo eléctrico, en el gas hay un equilibrio en el cual la rata de producción de partículas cargadas es igual a la rata de recombinación. Ahora, si colocamos dos electrodos, y a través de ellos colocamos una tensión DC, que iremos aumentando muy lentamente, se presenta una corriente debida al movimiento de los iones y electrones ya existentes; si la corriente es muy pequeña, no afectaría el equilibrio del gas, y su valor solo dependería de la velocidad con la cual los iones y los electrones llegan a los electrodos; así, esta aumenta casi linealmente con el potencial aplicado [3].

Aumentando más el potencial aplicado, podemos romper el equilibrio, debido a la cantidad de electrones e iones que llegan a los electrodos y son neutralizados, aumentando la rata de recombinación y reduciendo el número de partículas cargadas en el gas. Bajo esta situación, el incremento de la corriente con el potencial decrece gradualmente hasta el punto en el cual el número de partículas cargadas producidas por el gas es igual al número que llega a los electrodos; a partir de este momento nos encontramos en la región de saturación, en la cual la corriente de la descarga es independiente del potencial aplicado, como se muestra en la figura 1 para neón a 1 torr de presión, con electrodos circulares de $2 \mathrm{~cm}$ de diámetro, separados $50 \mathrm{~cm}$ [4].
Continuando a lo largo de la región de saturación, con aumentos sucesivos del potencial, llegaremos a un incremento exponencial de la corriente con la tensión, debida a la presencia de electrones con energía suficiente para producir nuevos iones y electrones por colisión con los átomos neutros. Además, si el potencial es lo suficientemente alto, los iones son acelerados hacia el cátodo, chocando con él y extrayendo electrones secundarios de su superficie [5]. Aumentos adicionales del potencial incrementarán la producción de cargas por choques de electrones con los átomos neutros del gas y por emisión secundaria de los electrodos, lo cual lleva a un incremento sobreexponencial de la corriente, seguido de un colapso del potencial a través de la descarga. Esta repentina transición se denomina ruptura, y se caracteriza por incrementos de varios órdenes de magnitud en la corriente con casi ningún incremento en el potencial.

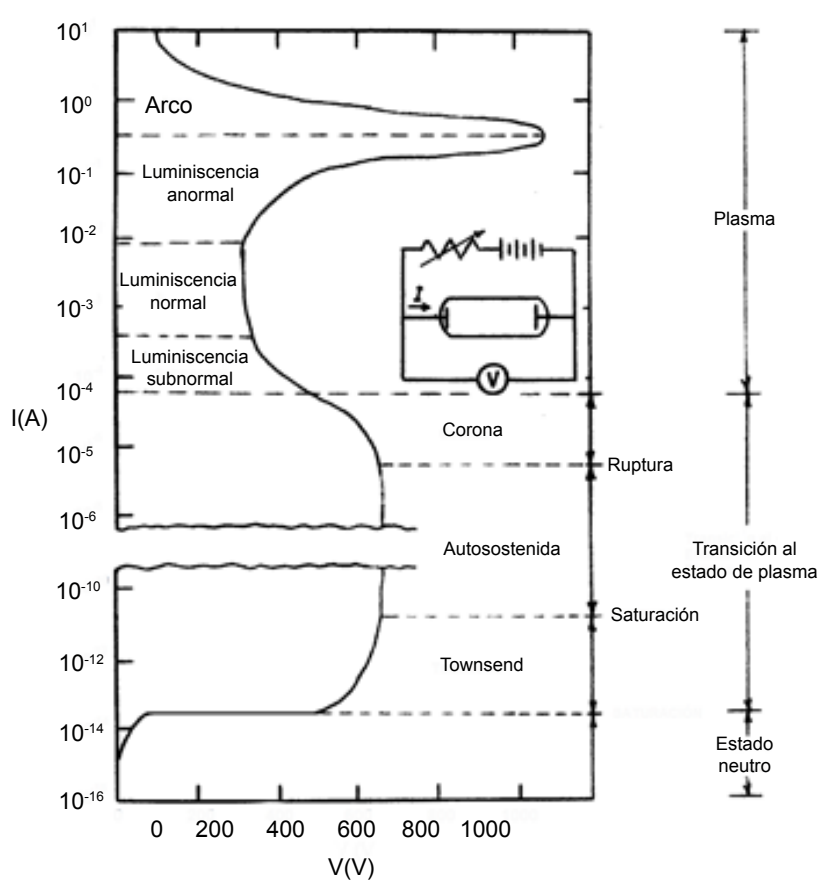

Figura 1. Relación Corriente-Voltaje para neón a 1 torr de presión con electrodos circulares de $2 \mathrm{~cm}$ de diámetro, separados $50 \mathrm{~cm} \mathrm{[4].}$ 
A partir de la ruptura tenemos una descarga autosostenida, pues el número de electrones secundarios producidos en el cátodo es suficiente para mantener la descarga, que, además, empieza a hacerse visible. La corriente continuará creciendo hasta llegar a la región de la descarga en corona, en la cual se forma una carga espacial responsable de la presencia de la no homogeneidad en el campo; esta descarga también es visible [6]. Posteriores incrementos de la corriente ocasionan una reorganización de los portadores de carga [7] y una disminución del voltaje a través de la descarga hasta alcanzar un nivel bajo a partir del cual la corriente aumentaría sin que haya cambios importantes en el potencial; estas regiones se conocen como regiones de luminiscencia subnormal y normal, respectivamente (ver figura 1). Enseguida, de estas dos regiones se presenta un nuevo incremento de la corriente en forma exponencial; a esta región se le denomina luminiscencia anormal [4]. Finalmente, más incrementos de la corriente nos permiten hablar de una nueva forma de descarga, conocida como descarga de arco, debido a su intenso brillo. En esta región vienen a ser importantes los efectos de la temperatura (ionización térmica) y la recombinación en el volumen del gas [8]. En la figura 1 se muestra el comportamiento de la corriente en un gas en función del potencial aplicado y las diferentes regiones de descarga [9].

El dispositivo diseñado para este trabajo, el cual se describe en la sección siguiente, trabaja en el régimen de la descarga luminiscente, y su comportamiento $\mathrm{V}$ vs. I será estudiado para diferentes atmósferas tanto en corriente continua como en corriente continua pulsada.

\section{Experimental}

Se diseñó un reactor tipo calorímetro (figura 2), constituido por dos electrodos cilíndricos concéntricos fabricados en acero inoxidable, cerrados por uno de sus extremos y acoplados entre sí por un material aislante (2), sellado con resina epóxica para conservar el vacío. El cilindro interno (1), de $38,8 \mathrm{~mm}$ de diámetro y 71,9 mm de altura, hace las veces de cátodo, donde se coloca cierta cantidad de agua ( 80 ml) para evitar el sobrecalentamiento del sistema, y cuya temperatura se observa con el termómetro (4). El electrodo externo (6) es el ánodo de la descarga, y sus dimensiones son $82,8 \mathrm{~mm}$ de diámetro y 73,5 mm de altura. El espacio comprendido entre el ánodo y el cátodo constituye la cámara de vacío y contiene el gas a baja presión a través del cual se genera la descarga eléctrica. La línea de vacío consta de una bomba mecánica de dos etapas (7), la cual se conecta a la cámara de vacío a través de la válvula de vacío (8). La válvula de vacío permite aislar o conectar la bomba mecánica a la cámara de vacío. Para la medida y monitoreo de la presión se utiliza un medidor de presión absoluta (9) de tipo capacitivo en el rango de 0 hasta 760 torr. La entrada de los gases (10), Aire, Ar e $\mathrm{H}_{2}$, a la cámara de vacío, regulada por los reguladores de flujo (11), se hace a través de la válvula de paso (12) acoplada al material aislante existente entre los dos electrodos.

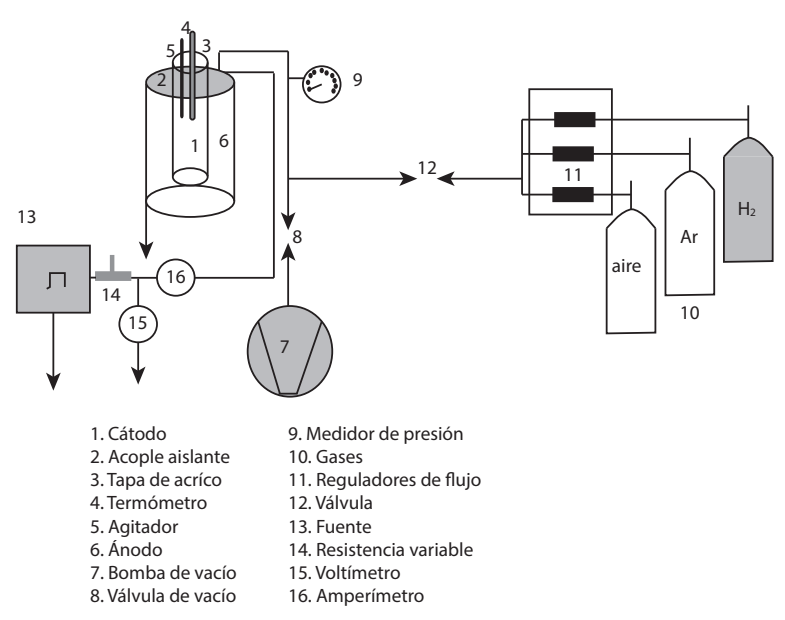

Figura 2. Reactor tipo calorímetro.

La potencia eléctrica se suministrará a la descarga por una fuente de corriente continua pulsante (13) de amplitud de voltaje variable desde 0 hasta $1000 \mathrm{~V}$. $\mathrm{El}$ acople entre la fuente y la descarga fue realizado a través de una resistencia (14) de 300 ohmios. El voltaje y la corriente de la descarga fueron medidos a través del voltímetro (15) y del amperímetro (16). Se observó el comportamiento de la descarga para dos tipos de corriente: corriente continua pulsante y corriente continua; para establecer esta última se conectó un banco de condensadores electrolíticos de $470 \mu \mathrm{F}$ a la salida de los rectificadores de la fuente de poder.

Después de configurado el reactor tipo calorímetro, se realizaron las pruebas preliminares tendientes a verificar el correcto funcionamiento de cada uno de sus subsistemas; para ello se encendió la descarga en una atmósfera de prueba (Aire), realizándose un 


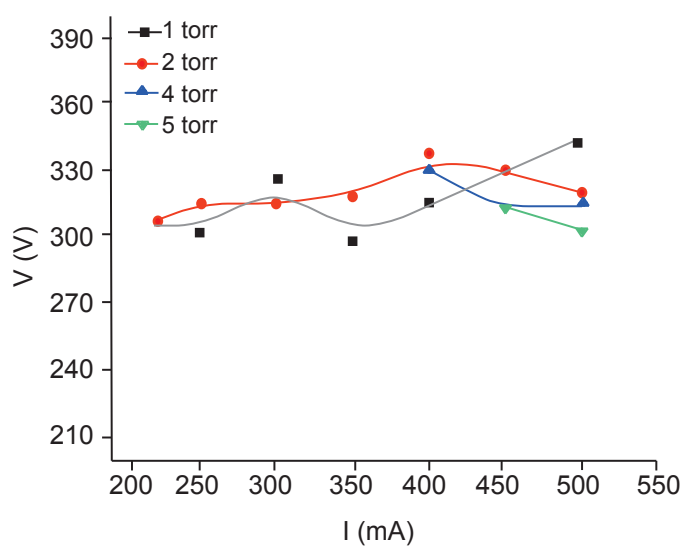

(a) Aire

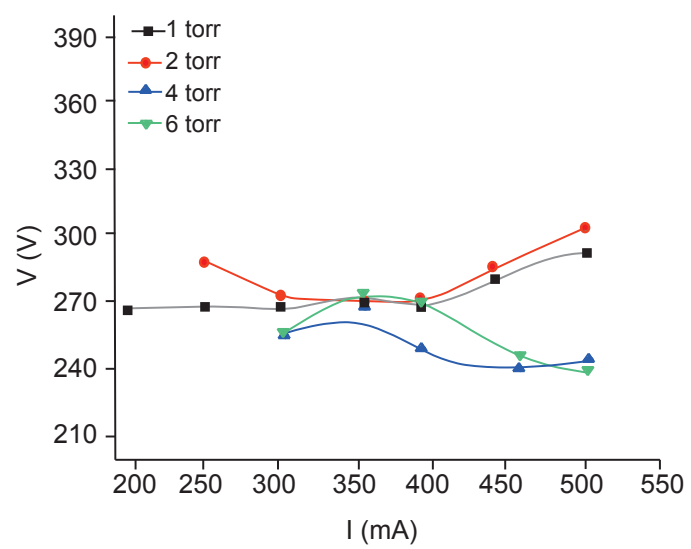

(b) $\mathrm{Ar}$

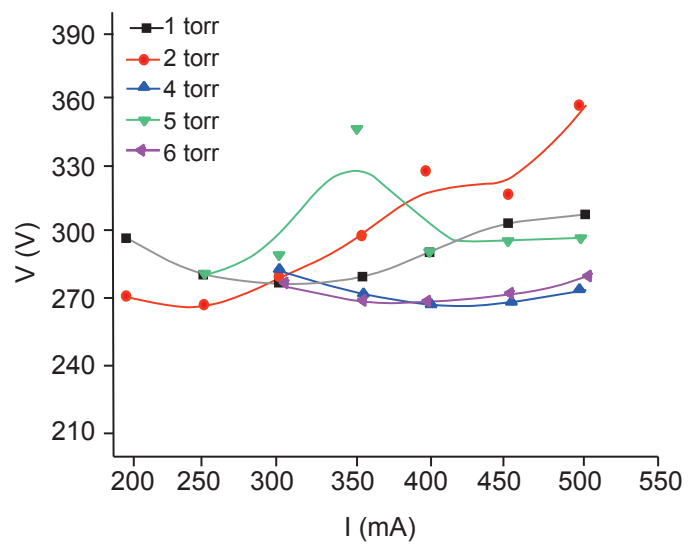

(c) $3 \mathrm{H}_{2}$

Figura 3. I vs. V para diferentes presiones con corriente continua pulsante, después de $60 \mathrm{~s}$.

barrido de la temperatura en función del voltaje de la fuente de poder; esto permitió observar el funcionamiento simultáneo de cada uno de los sistemas y realizar los ajustes definitivos necesarios para un normal funcionamiento del reactor en su conjunto, además de verificar el rango de temperatura en la cual no hubiese ablandamiento de la resina de sellado de la cámara y la consecuente presencia de fugas.

La incidencia de la presión en la curva V vs. I fue observada para los diversos gases utilizados (Aire, Argón e Hidrógeno), en el rango de 1 torr hasta 6 torr, estableciéndose las mejores condiciones de encendido de la descarga. También se observó la evolución en el tiempo, hasta los 90 s, de la curva I vs. $\mathrm{V}$, encendiendo la descarga y dejando los parámetros de encendido fijos. Otro comportamiento estudiado en las curvas I vs. V fue la incidencia del tipo de corriente, continua o continua pulsante. Es importante mencionar que cada curva fue realizada iniciando con el reactor, y el agua contenida en él, a tempera- tura ambiente. Las características I vs. V obtenidas fueron comparadas con la figura 1, estableciéndose el régimen de la descarga luminiscente generada para las diferentes atmósferas gaseosas empleadas en el dispositivo diseñado.

\section{Resultados y discusión}

La figura 3 muestra la relación I vs. V para cada uno de los gases con corriente continua pulsada después de 60 segundos de encendida la descarga a diferentes presiones, con $80 \mathrm{ml}$ de agua en el cátodo, a temperatura ambiente para evitar su sobrecalentamiento y una posible pérdida del vacío. Todas estas mediciones se realizaron en las mismas condiciones; se seleccionó un tiempo fijo, debido a que el voltaje varía levemente con el tiempo para una corriente fija.

En la figura 4 se muestra el comportamiento de la variación del voltaje en el tiempo para una presión de 2 torr en corriente continua. Comparando las curvas de la Figura 3 con la figura 1 [9], se puede establecer que para la mayoría de las condiciones 


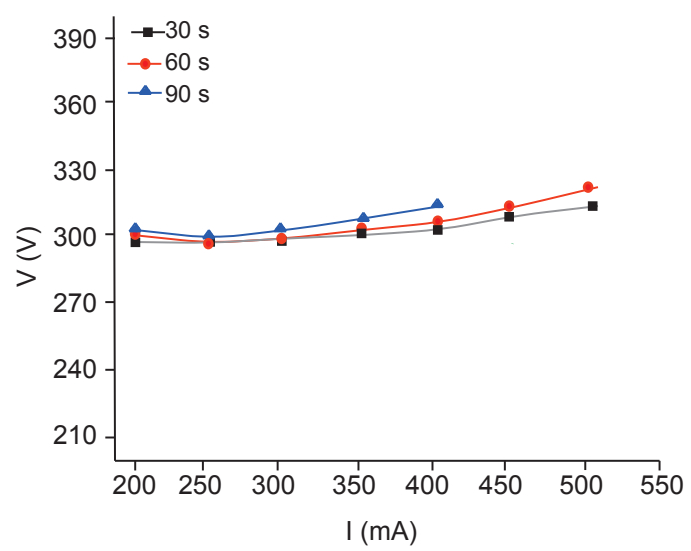

(a) Aire

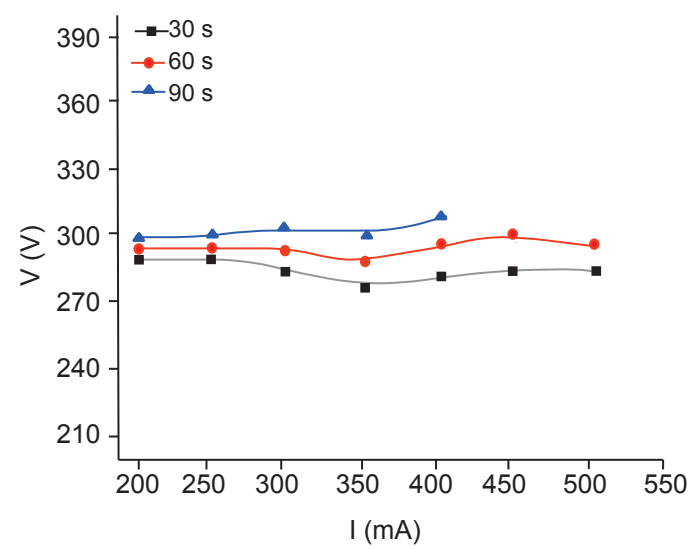

(b) $\mathrm{Ar}$

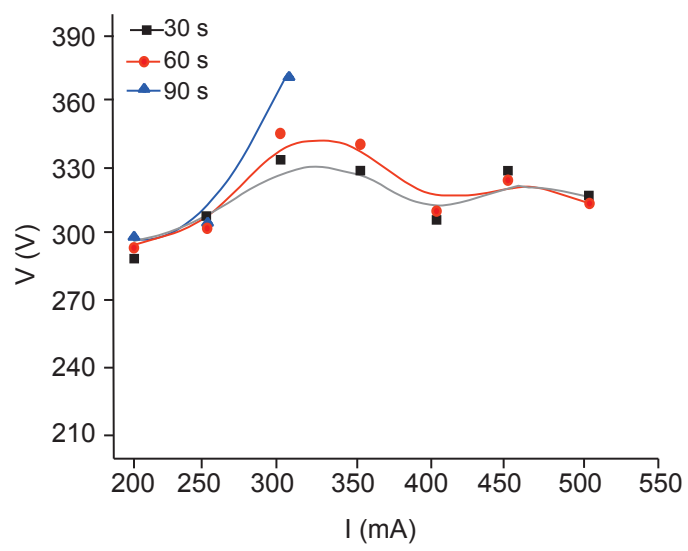

(c) $3 \mathrm{H}_{2}$

Figura 4. I vs. V a 2 torr para diferentes tiempos con corriente continua.

de presión y atmósferas utilizadas en el reactor, la descarga establecida se encuentra en el régimen normal y subnormal de la descarga luminiscente $[4,6$, 8]. Solo para las presiones superiores a 4,0 torr, inclusive para aire y argón se observa una marcada disminución del voltaje aplicado mientras la corriente aumenta, lo cual es característico de la descarga luminiscente subnormal (ver figura 1). Para aire a presiones de 4 y 5 torr solo consigue iniciarse la descarga a partir de $400 \mathrm{~mA}$ y $450 \mathrm{~mA}$. Considerando el tipo de gas utilizado, pueden observarse potenciales menores para mantener la descarga, bajo las condiciones estudiadas, en el gas más pesado (Ar, figura $3 b)$, lo cual puede explicarse por su mayor eficiencia en la generación de electrones secundarios por impacto sobre el cátodo, mecanismo muy importante para la generación y sostenimiento de la descarga luminiscente $[2,8]$.

En las curvas de la figura 4 puede observarse un ligero incremento de la tensión en el tiempo, que puede deberse al progresivo calentamiento del cátodo, lo cual disminuye la densidad del medio gaseoso en su proximidad, requiriéndose de una mayor diferencia de potencial en la descarga para compensar la disminución de corriente por disminución de la densidad del gas. También se observa una mayor diferencia de potencial para mantener la descarga en el gas más pesado (Ar) como fue discutido en el párrafo anterior. Para el tiempo de 90 segundos se hace un barrido hasta valores de voltaje en los cuales el agua contenida en el cátodo alcanza su punto de ebullición, lo cual puede ocasionar pérdida de masa del sistema. 


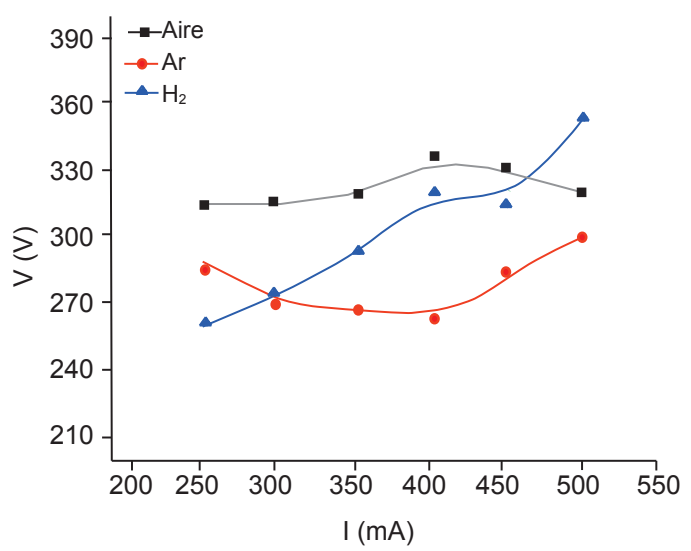

(a) Con corriente continua pulsante

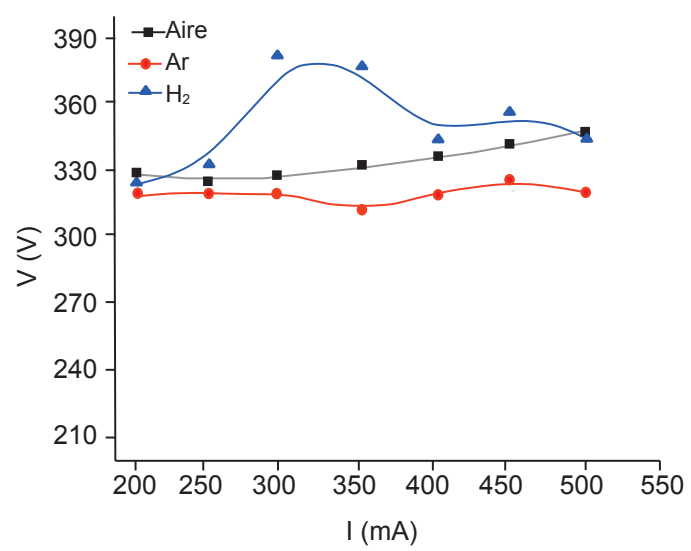

(b) Con corriente continua

Figura 5. I vs. V para $60 \mathrm{~s}$ a 2 torr con diferente gas.

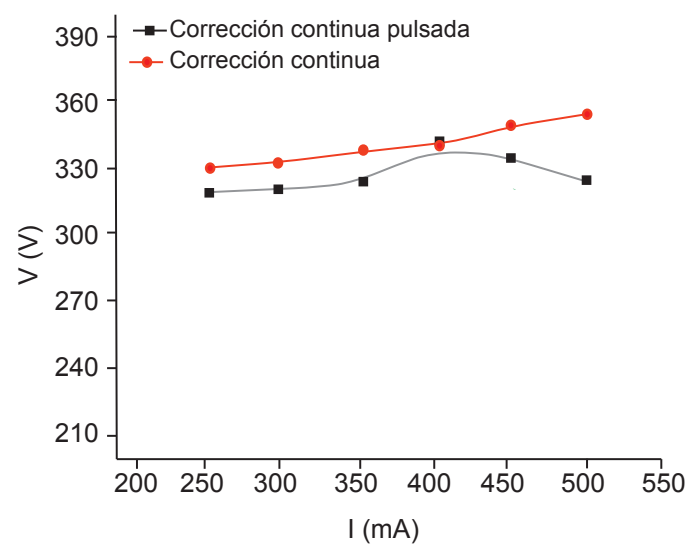

(a) Con corriente continua

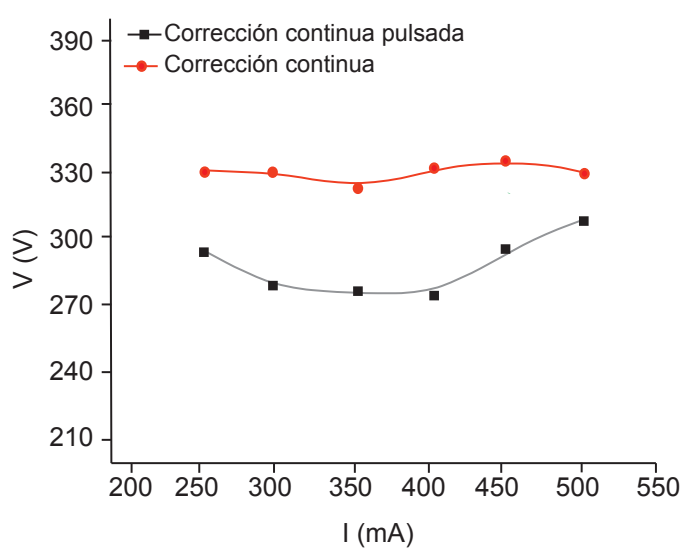

(d) Con corriente continua

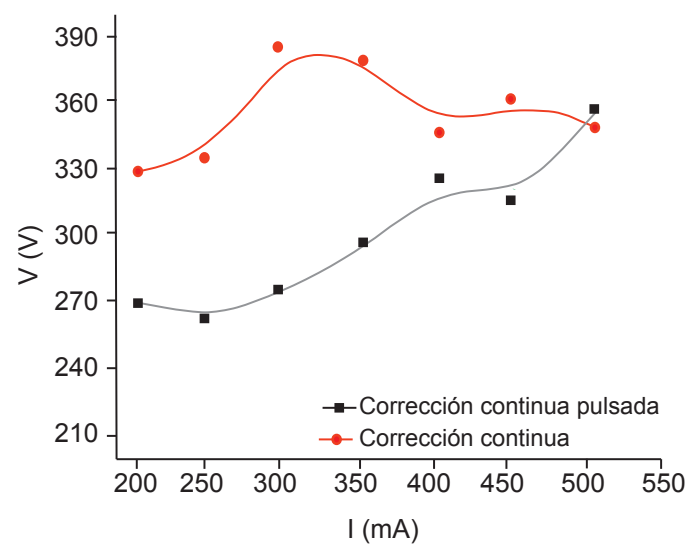

(c) Con corriente continua

Figura 6. I vs. V para $60 \mathrm{~s}$ a 2 torr con corriente continua pulsada y corriente continua.

En la figura 5 se encuentra la relación I vs. V para una presión de 2 torr con corriente continua pulsante y corriente continua para los diferentes tipos de gas, tomando el voltaje después de $60 \mathrm{~s}$ de iniciada la descarga; cada medida (cada punto de la curva) fue iniciada con la misma cantidad de agua $(80,0 \mathrm{ml})$ en el reactor y a la temperatura ambiente; la presión de
2 torr fue elegida con base en la mejor respuesta de calentamiento observada en el cátodo.

En la figura 5a, para corriente continua pulsante se encuentra un comportamiento de descarga luminiscente normal, o anormal con una pendiente muy pequeña, para el Aire y el Ar, requiriéndose de 
menos voltaje para el gas más pesado; para el $\mathrm{H}_{2}$ se observa un comportamiento característico de descarga luminiscente anormal [9] bajo estas condiciones. El hidrógeno presenta una tendencia lineal de I vs. $\mathrm{V}$ que indica que, por su carácter más leve, obedece más fácilmente a las variaciones del voltaje aplicado, variando casi linealmente su eficiencia para generar electrones secundarios por impacto sobre el cátodo.

En la figura 5b, para corriente continua se tiene que para Ar el voltaje para mantener la descarga es menor al voltaje empleado para mantener la misma descarga a una misma corriente utilizando Aire $\mathrm{o}_{2}$, respectivamente. Aunque para establecer corrientes por debajo de los $250 \mathrm{~mA}$ la diferencia entre los voltajes es muy pequeña, para establecer corrientes por encima de los $300 \mathrm{~mA}$ se requiere de diferencias de voltaje más amplias; este comportamiento es más evidente para $\mathrm{H}_{2}$, donde se ve su mayor incremento, comparado con los otros dos gases; esto se debe a que el $\mathrm{H}_{2}$ es más liviano, por lo que sus iones tienden a extraer menos partículas por impacto sobre el cátodo, y requiere de más potencial para mantener la descarga; lo contrario pasa con Ar, que al ser el gas más pesado requiere de menos potencial para mantener la descarga.

En la figura 6 se muestra la comparación, para cada uno de los gases, de las curvas I vs. V realizadas en corriente continua pulsante y en corriente continua, a presión de 2 torr, con un tiempo de medida, después de encendida la descarga, de 60 segundos. Puede observarse que se requiere de un menor valor en el voltaje con corriente continua pulsante para mantener la descarga con la misma corriente, con relación al tipo de descarga con corriente continua; esto se debe a que el voltaje correspondiente observado por los iones cerca del cátodo en corriente continua pulsante es 1,41 veces el promedio indicado por el medidor, permitiendo inferir la presencia de partículas más energéticas incidiendo sobre el cátodo, en la descarga en corriente continua pulsante, lo cual representa una transferencia más eficiente de energía entre el cátodo y el plasma de la descarga luminiscente.

\section{Conclusiones}

La descarga luminiscente de baja presión generada a través de aire, argón e hidrógeno, y en regímenes de corriente continua y corriente continua pulsante, mediante un dispositivo tipo calorímetro, se encuentran en la región de las descargas luminiscente normal y anormal descritas en la curva I vs. V, siendo predominantemente la descarga luminiscente normal la que se desarrolla en las atmósferas gaseosas con átomos de mayor número atómico, como el Ar y el Aire, mientras que en atmósferas con menor número atómico, como la de $\mathrm{H}_{2}$, se generan descargas luminiscentes típicamente anormales.

\section{Referencias}

[1] G. G. Bondarenko, V. I. Kristya, M. I. Supelnyak, "Calculation of the electrode surface temperature in the normal glow discharge". Journal of Vacuum, vol. 86, p. 854-856, 2012.

[2] B. Chapman, Glow Discharge Processes. New York: John Wiley and Sons, 1980.

[3] M. M. Abdel Rahman, A. Helal, O. A. Moustafa, and F. W. Abdel Salam, "High Efficiency Glow Discharge Ion Source". Journal of $\mathrm{Nu}$ clear and Radiation Physics, Vol. 3, no. 1, pp. 1-9, 2008.

[4] N. Essam, Fundamental of Gaseous Ionization and Plasma Electronics. New York: John Wiley, 1971.

[5] A. Sarmiento Santos, Introdução de Nitrogênio em Ligas Sinterizadas de $\mathrm{Fe}$-Cr, Submetidas a uma Descarga Luminescente Anormal. Tesis de doctorado, UFSC, Florianópolis, Brasil, 2003.

[6] V. Vicuña Hernández, Análisis Espectroscópico y Caracterización de un Plasma de Resplandor, Tesis de pregrado, UNA, México, 2008.

[7] A. M. Howatson, An Introduction to Gas Discharges. Oxford: Pergamon Press, 1976.

[8] A. Von Engel, Ionized Gases, Oxford: Pergamon Press, 1996.

[9] C. E. Rojas Sánchez, Implementación y evaluación de un sistema para tratamiento de materiales usando descarga luminiscente anormal, Tesis de maestría, Uptc, Tunja, 2010. 